\title{
Composition corporelle de poulets «Cou nu " ou normalement emplumés selon le taux protéique de la ration
}

\author{
A. ZEIN-EL-DEIN (1), P. MÉRAT et A. BORDAS \\ I.N.R.A., Laboratoire de Génétique factorielle, \\ Centre de Recherches zootechniques, F 78350 Jouy-en-Josas
}

\begin{abstract}
Résumé
Des poulets hétérozygotes pour le gène «Cou nu " $\left(\mathrm{Nana}^{+}\right)$ou à plumage normal $\left(n a^{+} n a^{+}\right)$, au nombre de 119 au total, ont été répartis dans 2 lots recevant un aliment à $2900 \mathrm{kcal} \mathrm{E}$.M. $/ \mathrm{kg}$ et à taux protéique différent (respectivement 16 et 20 p. 100) ; les mâles étaient élevés en cages individuelles et les femelles au sol. La température ambiante après 4 semaines était maintenue à $29{ }^{\circ} \mathrm{C}$ pour les mâles et environ $24^{\circ} \mathrm{C}$ pour les femelles. L'abattage avait lieu à 75 et 82 jours respectivement pour les coquelets et les poulettes. Les 2 génotypes ont été comparés séparément dans chaque sexe pour la composition des carcasses. Dans tous les cas, le génotype $\mathrm{Na} \mathrm{na}^{+}$avait moins de pertes après plumage, un rendement en viande supérieur dans la carcasse éviscérée, un pourcentage de tissus gras plus faible (significativement seulement pour le gras intermusculaire). Le régime le plus riche en protéines totales s'accompagnait d'un engraissement moindre. Il n'y avait pas, dans l'ensemble, d'interactions entre génotype et régime pour les paramètres étudiés.
\end{abstract}

Mots clés : Poulet, composition corporelle, Cou nu, aliment, protéine.

\begin{abstract}
Summary
Body composition of " naked neck" vs normally-feathered chickes fed with two different dietary protein levels
\end{abstract}

A total of 119 chickes heterozygous for the "naked neck" gene $\mathrm{Nana^{+ }}$ or with normal plumage $\mathrm{na}^{+} \mathrm{na}^{+}$were divided into 2 groups and fed a diet with $2900 \mathrm{kcal}$ M.E. $/ \mathrm{kg}$ and protein levels of 16 and 20 p. 100, respectively. The males were raised in individual cages and the females on floor. After 4 weeks, ambient temperature was maintained at $29{ }^{\circ} \mathrm{C}$ for the males and at about $24^{\circ} \mathrm{C}$ for the females. The males were slaughtered at 75 days of age and the females at 82 days. The carcass composition of the 2 genotypes was compared separately in each sex. In all cases, the $\mathrm{Na} \mathrm{na}{ }^{+}$genotype showed a lower loss after defeathering, a higher meat yield of the eviscerated carcass and a lower percentage of fatty tissue (significant only for intermuscular fat). The diet with the highest total protein content resulted in the least fat content. With few exceptions, there was no genotype-diet interaction for the traits under study.

Key words : Broiler, body composition, naked neck, feed, protein.

(1) Adresse permanente : Département de Production animale, Faculté d'Agriculture, Université Aïn-Shams, Le Caire, Egypte. 


\section{Introduction}

Le gène " Cou nu » $(\mathrm{Na})$ a une influence favorable sur la croissance pondérale de poulets à une température supérieure à $30^{\circ} \mathrm{C}$ (BORDAS et al., 1978 ; MONNET et al., 1979 ; HANZl \& Somes, 1983), ou en saison chaude en Egypte (ZEIN-EL-DeIN et al., 1981a).

Quant à la composition corporelle, une réduction du poids du plumage de l'ordre de $30 \mathrm{p} .100 \mathrm{chez}$ l'hétérozygote $\mathrm{Na} \mathrm{na}{ }^{+}$et $40 \mathrm{p} .100 \mathrm{chez}$ l'homozygote a été observée (Bordas et al., 1978 ; MONNET et al., 1979). D'autre part, selon ZEIN-EL-DeIN et al. (1981b), des poulets "Cou nu » hétérozygotes présentaient dans les 2 sexes une proportion plus élevée de muscles par rapport au squelette et un meilleur rendement en viande de la carcasse éviscérée que des poulets normalement emplumés de même origine.

Nous avons voulu dans le présent travail confirmer ce résultat sur des effectifs plus importants, dans la dernière phase d'une expérience relatée par ailleurs (ZEIN-EL-DEIN et al., 1984) où des poulets de génotype $\mathrm{Nana^{+ }}$ et $n a^{+} n a^{+}$étaient répartis dans 2 lots recevant 2 taux protidiques différents dans la ration. L'objet était, comme indiqué dans l'article cité, de savoir si des poulets «Cou nu » pouvaient être relativement plus avantagés en présence d'un aliment à taux protéique inférieur aux normes habituelles.

\section{Matériel et méthodes}

\section{A. Génotypes}

Les poulets sont issus du croisement de 9 coqs normalement emplumés $\left(n a^{+} n a^{+}\right)$ avec des poules hétérozygotes $\mathrm{Na} \mathrm{na}{ }^{+}$. A l'éclosion (octobre 1982), le sexe et le génotype $\left(\mathrm{Nana^{+ }}\right.$ ou na+ $\left.n a^{+}\right)$étaient déterminés et chaque poussin bagué.

\section{B. Conditions expérimentales}

Elles sont décrites en détail par ailleurs (ZEIN-EL-DEIN et al., 1984). Nous rappellerons les points essentiels. Les 2 sexes étaient traités de façon séparée. Les femelles étaient élevées au sol jusqu'à l'abattage à une température moyenne comprise entre 23 et $24{ }^{\circ} \mathrm{C}$ à partir de l'âge de 4 semaines. Quant aux mâles, 60 étaient gardés par génotype, choisis au hasard dans les familles les plus nombreuses, et placés à partir de l'âge de 1 semaine dans un local maintenu à $29^{\circ} \mathrm{C} \pm 1^{\circ} \mathrm{C}$. Tous les poussins recevaient $10 \mathrm{~h}$ de lumière par jour. Dans les 2 sexes, chaque génotype était réparti par moitié en 2 lots, recevant respectivement une ration à 16 et 20 p. 100 de protéines totales de l'éclosion à l'abattage. Les 2 rations contenaient approximativement $2900 \mathrm{kcal} / \mathrm{kg}$ d'énergie métabolisable. Le tableau 1 détaille la composition des 2 régimes.

L'abattage avait lieu à 75 et $82 \mathrm{j}$ respectivement chez les coquelets et les poulettes. Les mesures de carcasse étaient faites sur 15 poulets par génotype et régime dans chaque sexe (à l'exception des mâles $n a^{+} n a^{+}$en régime riche au nombre de 14), soit 119 au total. Dans chaque sexe et chaque régime, les individus $\mathrm{Na} \mathrm{na}^{+}$et $\mathrm{na}^{+} \mathrm{na}^{+}$ étaient choisis par couples de frères ou demi-frères et sœurs. 


\section{TABLEAU 1}

Composition des régimes.

Composition of the feeds.

\begin{tabular}{|c|c|c|}
\hline \multirow{2}{*}{ Composants } & \multicolumn{2}{|c|}{ p. 100 du total } \\
\hline & Régime 1 & Régime 2 \\
\hline 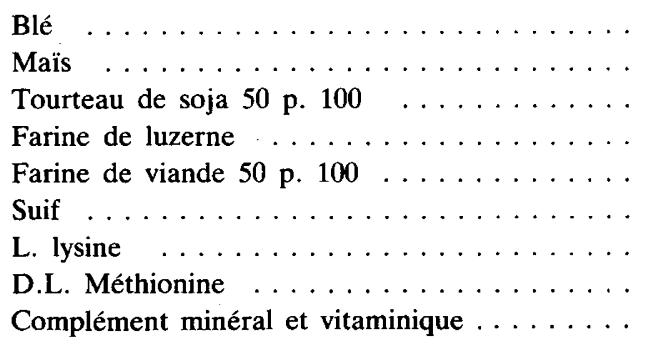 & $\begin{array}{l}40,00 \\
39,40 \\
10,90 \\
1,10 \\
5,50 \\
- \\
0,182 \\
- \\
2,91\end{array}$ & $\begin{array}{l}40,00 \\
33,50 \\
16,24 \\
- \\
8,00 \\
0,30 \\
- \\
0,098 \\
1,86\end{array}$ \\
\hline \multicolumn{3}{|l|}{ Principales caractéristiques } \\
\hline $\begin{array}{l}\text { Protéines totales, p. } 100 \ldots \ldots \ldots \\
\text { Energie métabolisable, } \mathrm{kcal} / \mathrm{kg} \quad \ldots \ldots \ldots \ldots \\
\text { Calcium, p. } 100, \ldots \ldots \ldots \ldots \\
\text { Phosphore total, p. } 100 \ldots \ldots \ldots \ldots \ldots \ldots \ldots\end{array}$ & $\begin{array}{l}16,0 \\
2900 \\
1,0 \\
0,6\end{array}$ & $\begin{array}{l}20,0 \\
2900 \\
1,0 \\
0,6\end{array}$ \\
\hline
\end{tabular}

\section{Mesures}

Parmi les données relatives à la croissance et à la consommation alimentaire, présentées par ailleurs (ZEIN-EL-DEIN et al., 1084), l'indice de consommation de 4 à 10 semaines des mâles est pris en compte ici pour ses corrélations avec les variables de composition corporelle.

Les autres mesures prises figurent aux tableaux 2 et 3 . Leur définition appelle les précisions suivantes :

- Le poids vif est déterminé avant abattage, le matin, sur les oiseaux mis à jeun depuis $17 \mathrm{~h}$ la veille.

- Toutes les mesures sont exprimées en p. 100 du poids vif, à l'exception des poids de peau, d'os, de muscles et de graisse intermusculaire, qui sont calculés en p. 100 de la demi-carcasse éviscérée. Une variable dérivée des précédentes est le rapport viande/os.

- Le poids de sang et le poids de plumes sont déterminés par différence, respectivement après saignée et plumage à sec.

- La tête est séparée à la base de l'os occipital, les pattes à la jointure tibio-tarsale.

- La graisse abdominale est prélevée autour du gésier, de la bourse de Fabricius et sur la paroi abdominale, la graisse attachée à l'intestin étant exclue (RICARD \& 


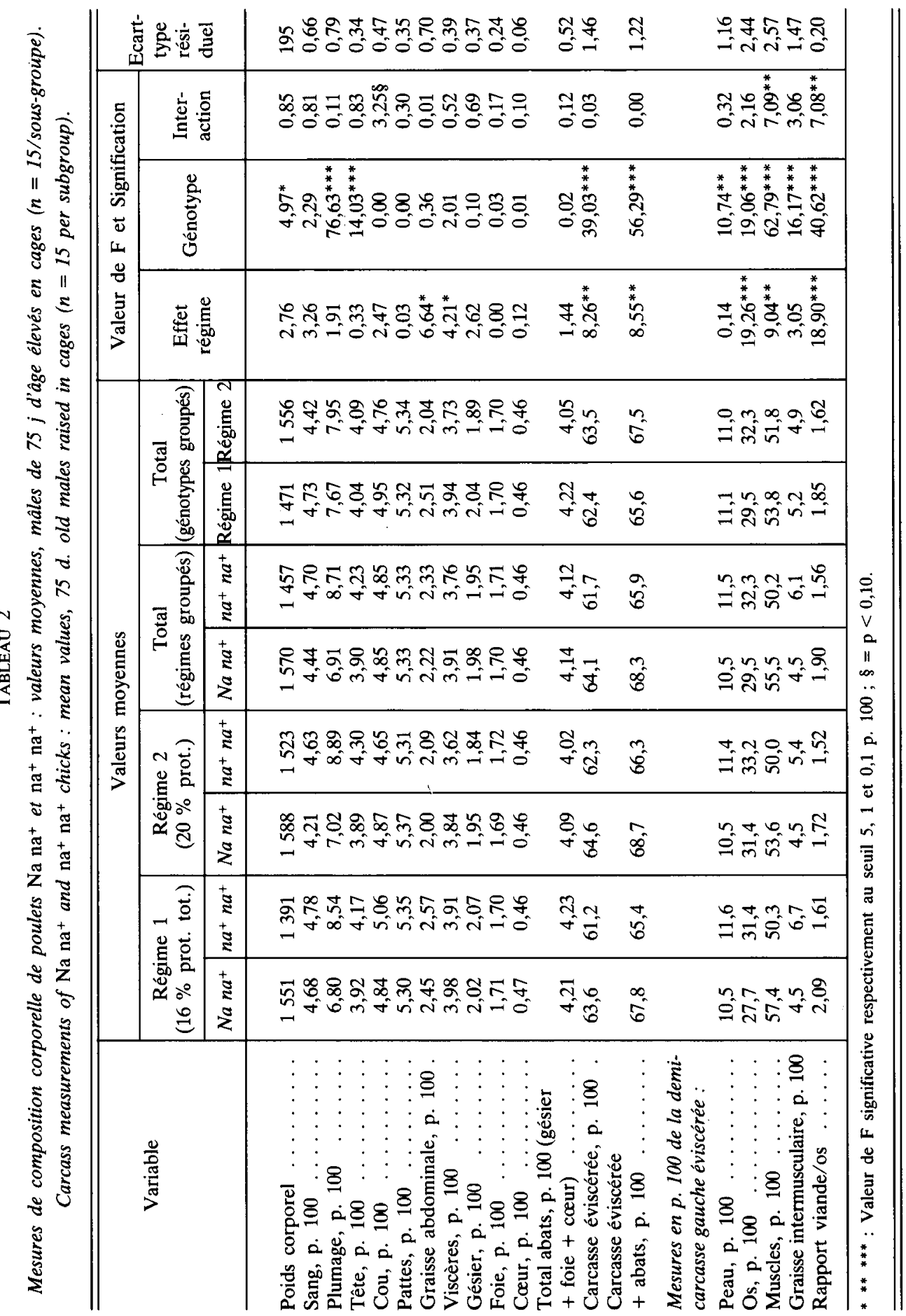




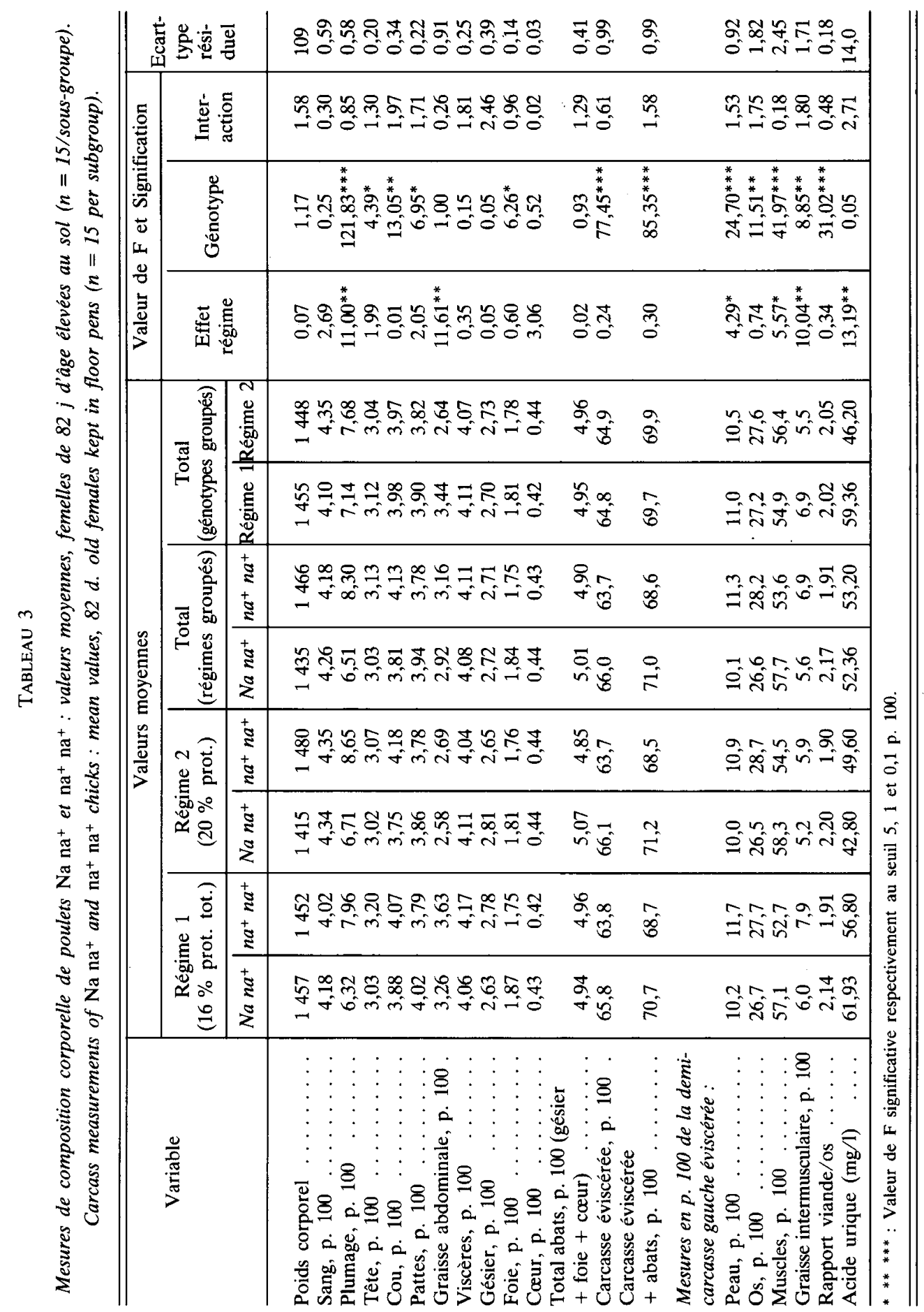


Rouvier, 1967 ; RiCARD, 1972). Ce prélèvement est fortement lié aux taux de lipides de la carcasse entière (DELPECH \& RICARD, 1965).

- La graisse intermusculaire comprend les dépôts gras situés entre les muscles et le long des vaisseaux, ainsi que les dépôts sous-cutanés. Le poids de la peau n'inclut donc pas ces derniers.

- Les viscères et le gésier sont pesés vides.

- Le poids de la carcasse éviscérée est compté après déduction du sang, plumes, tête, cou, pattes, ensemble des viscères et gras abdominal.

\section{Analyse statistique}

Les données recueillies respectivement sur les mâles et sur les femelles sont considérées comme 2 séries expérimentales indépendantes, analysées de façon séparée. Pour chaque caractère et dans chaque sexe, l'analyse de variance comporte les facteurs " régime » et " génotype » et leur interaction.

Les corrélations phénotypiques sont présentées pour 12 variables de composition corporelle. Chez les mâles, l'indice de consommation de 4 à 10 semaines leur est ajouté. Le détail des corrélations à l'intérieur de chaque régime et génotype n'est pas présenté ; un test d'homogénéité des corrélations entre sous-groupes n'ayant révélé une hétérogénéité significative que dans un petit nombre de cas, une estimation de chaque corrélation regroupée après transformation (variable $Z$ ) sur une base intra-groupe est donnée. Dans les cas d'hétérogénéité, cette valeur regroupée apparaît entre parenthèses à titre indicatif.

\section{Résultats et discussion}

\section{A. Valeurs moyennes}

Le dispositif expérimental ne permet pas de discuter de l'effet du sexe sur les variables étudiées. Par contre, dans la mesure où les résultats obtenus dans chaque sexe présentent des analogies, ils seront rapprochés pour leur discussion.

Les tableaux 2 et 3 donnent respectivement chez les mâles et les femelles les valeurs moyennes pour les génotypes $\mathrm{Nana^{+ }}$ et $\mathrm{na}^{+} \mathrm{na}^{+}$en présence de chaque taux protéique, les moyennes générales par génotype (régimes groupés) et par régime (génotypes groupés), et le test de signification des effets « génotype » et.« régime » et de leur interaction.

\section{B. Effets du génotype}

L'effet "génotype » sur le poids corporel a déjà été discuté sur l'effectif total de la même expérience (ZEIN-EL-DEIN et al., 1984). Quant à la réduction hautement significative du poids relatif du plumage par le gène $\mathrm{Na}$ dans les 2 sexes, elle confirme des observations antérieures (BORDAS et al., 1978 ; MONNET et al., 1979).

A part la proportion un peu moindre du cou et plus grande des pattes vis-à-vis du poids vif pour les poulets «Cou nu » dans le sexe femelle, non expliquées, 2 groupes 
d'effets associés au génotype ressortent de l'ensemble des résultats, relatifs à la proportion d'os par rapport aux muscles, et à la proportion de tissus gras.

Dans la demi-carcasse éviscérée, le pourcentage d'os est partout inférieur et celui de muscles supérieur pour les animaux $\mathrm{Nana^{+ }}$ par comparaison à leurs homologues à emplumement normal. L'effet " génotype » est hautement significatif dans chaque sexe, ainsi que pour le rapport viande/os. Peut-être l'importance proportionnellement un peu plus faible de la tête chez les oiseaux "Cou nu ", significative également dans les 2 sexes, correspond-elle au même effet, la tête comportant une grande part de squelette. La proportion relative d'os n'avait pas été trouvée différente entre les génotypes $\mathrm{Na} \mathrm{na}{ }^{+}$ et $n a^{+} n a^{+}$par Bordas et al. (1978), en ce qui concerne la cuisse et le pilon. La différence trouvée ici dans l'ensemble de la carcasse pourrait donc se localiser dans le tronc. L'importance comparée des muscles pectoraux serait à vérifier dans les génotypes étudiés. A propos du gène «Scaleless " d'effet analogue à $\mathrm{Na}$ mais plus extrême quant à la réduction du plumage, SOMES \& JOHNSON (1982) signalent qu'une meilleure conformation de la poitrine lui est associée.

L'avantage des animaux "Cou nu » pour la proportion de muscles ne dépend pas d'une différence dans le poids vif. Cette dernière n'est pas considérable, et de plus une analyse de covariance (non détaillée ici) du pourcentage de muscles ou du rapport muscles/os à poids corporel fixé ne change pas la signification des différences pour les deux premières variables.

L'interprétation de la croissance relativement plus rapide du tissu musculaire associée au gène $N a$ nécessiterait d'autres recherches. On peut suggérer que durant le développement du jeune, la formation d'une plus petite quantité de plumage laisse disponibles des matières protéiques pour d'autres tissus, dans la mesure où la rétention azotée ne diffère pas. D'autre part, GoLDSPINK (1977) indique que le nombre de fibres musculaires, qui est un des facteurs du développement ultérieur de la musculature, est déterminé durant la vie embryonnaire. Au cours de celle-ci, la répartition d'un apport protéique obligatoirement limité entre duvet et musculature pourrait être déplacée chez les embryons «Cou nu » en faveur du tissu musculaire.

L'autre groupe d'effets significatifs ou hautement significatifs concerne la proportion de graisse dans différentes régions corporelles. L'effet le plus marqué s'exerce sur la graisse intermusculaire de l'ensemble de la demi-carcasse, qui partout est en proportion inférieure chez les poulets "Cou nu ». Pour la graisse abdominale, les différences ne sont pas significatives, mais elles sont toujours de même sens. On peut suggérer la même interprétation pour le poids de la peau, inférieur pour le génotype $\mathrm{Nana}{ }^{+}$dans les 2 sexes et les 2 régimes, ce poids étant en corrélation positive avec l'engraissement (voir par exemple RICARD, 1968 ; EHINGER, 1977 ; MÉRAT et al., 1980). Au total, la réduction du tissu adipeux observée ici en présence du gène "Cou nu » concorde avec les observations de HANZL \& SOMES (1983), ainsi que de Bordas et al. (1978).

Enfin, la part toujours plus grande de la carcasse éviscérée par rapport au poids vif chez les oiseaux "Cou nu " correspond visiblement au fait que leur pourcentage de pertes après plumage est réduit.

Des tableaux 2 et 3 on peut conclure, sur l'ensemble des 2 régimes, à un gain de 2,4 p. 100 associé au génotype "Cou nu » hétérozygote quel que soit le sexe, sur le rendement à l'abattage (total de la carcasse éviscérée et des abats en p. 100 du poids vif). De plus, en estimant les rendements en viande à partir des mêmes tableaux, on constate que, pour la viande non dégraissée (carcasse désossée plus abats), son pourcen- 
tage par rapport au poids vif chez les poulets "Cou nu » dépasse celui obtenu chez les poulets $n a^{+} n a^{+}$respectivement de 3,4 et $2,8 \mathrm{p}$. $100 \mathrm{chez}$ les mâles et les femelles. Pour la viande dégraissée (graisse intermusculaire déduite), les gains correspondants sont respectivement 4,5 et 3,7 p. 100 .

\section{Interactions génotype $\times$ régime}

Quant aux interactions génotype $\times$ régime, on n'en trouve que pour 2 caractères, pourcentage de muscles et rapport muscles/os dérivant du précédent, et seulement chez les mâles, les différences entre génotypes étant plus accentuées avec un régime moins riche en protéines. Il est intéressant de noter que les principaux effets du gène «Cou nu » sont donc indépendants du taux protéique du régime, ou du rapport énergie/protéines.

\section{Effet du régime}

L'effet propre du régime est apparent sur le tissu gras et les plumes. La graisse abdominale est significativement réduite par le régime riche, ainsi que la graisse intermusculaire chez les femelles, la différence étant de même sens chez les mâles. L'effet sur la peau, par contre, n'apparaît que chez les femelles. Une influence sur l'engraissement du rapport des protéines à l'énergie dans la ration est connue : par exemple, KUIT (1962), Holshema (1975) cités par VAN MiddelKoOP et al. (1977) ; TouCHBURN et al. (1981) ; EhINGER \& SEEMANN (1982).

Le pourcentage de plumes est partout légèrement augmenté par le régime riche, significativement chez les femelles seules.

Les autres effets sont difficiles à interpréter, limités à un sexe (pourcentage de viscères, d'os, proportion de carcasse éviscérée), ou de sens contraire chez les mâles et les femelles (pourcentage de muscles).

\section{E. Corrélations}

Le tableau 4 présente dans chaque sexe les corrélations phénotypiques des variables de composition corporelle entre elles et avec le poids à l'abattage, auquel s'ajoute l'indice de consommation chez les mâles.

L'interprétation des cas, peu nombreux, d'hétérogénéité d'un coefficient de corrélation entre génotypes ou régimes est peu claire, compte tenu, de plus, de l'effectif limité dans chaque sous-groupe. Nous ne discuterons donc que les estimations réalisées sur l'ensemble des groupes, lorsqu'elles sont significativement différentes de zéro.

Les animaux les plus lourds tendent à être proportionnellement les plus gras : c'est ce que reflètent les corrélations significatives et positives du poids vif avec la proportion de graisse abdominale dans les 2 sexes, avec la graisse intermusculaire chez les femelles ; cette dernière corrélation n'est pas significative chez les mâles mais est positive également. La corrélation positive chez les femelles entre le poids corporel et le pourcentage de peau pourrait s'interpréter de même, compte tenu de la liaison trouvée dans la littérature (cf. plus haut) entre l'engraissement et le poids ou l'épaisseur de la peau. La tendance des animaux à croissance plus rapide à être plus gras est bien connue et a été souvent 


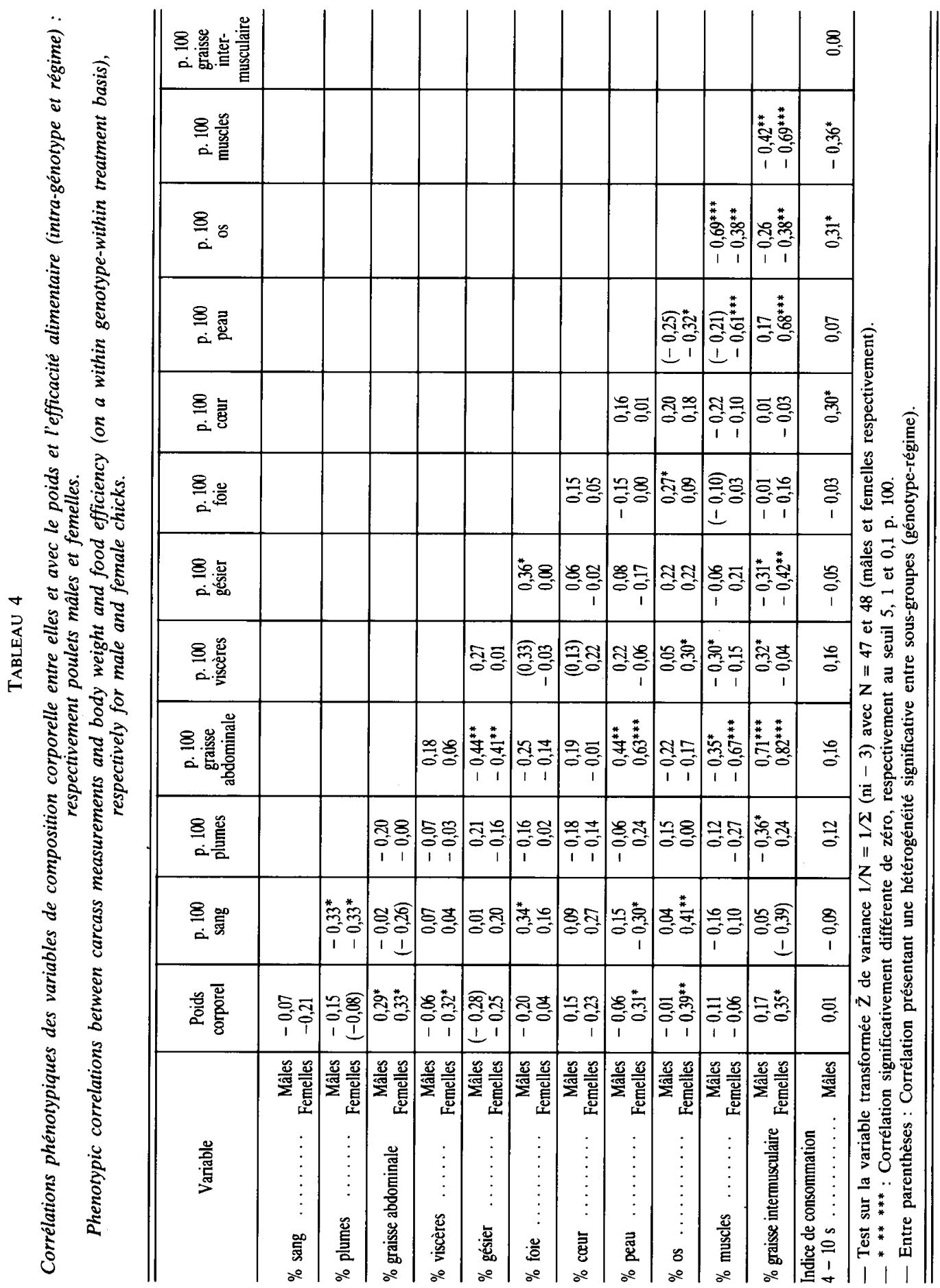


discutée (par exemple, revue par VAN MIDDELKOOP et al. (1977), LIN (1981)). On note encore, chez les femelles, une corrélation négative du poids corporel avec la proportion d'os et de viscères.

Pour l'indice de consommation, mesuré individuellement chez les mâles, on remarque sa corrélation positive avec le pourcentage d'os et négative avec le pourcentage de muscles dans la carcasse éviscérée, significative au seuil de $5 \mathrm{p} .100$ : les poulets moins osseux et plus musclés ont un meilleur indice. Par contre, on ne trouve pas de relation significative avec les variables mesurant l'engraissement, ce qui n'est pas conforme à la liaison négative généralement trouvée (par exemple, LECLERCQ et al., 1980 ; CHAMBERS et al., 1983).

Parmi les corrélations entre variables de composition corporelle, certaines proviennent de ce que plusieurs variables représentent des parties d'un même total : c'est le cas de la corrélation négative hautement significative dans les deux sexes entre pourcentage d'os et de muscles; on peut en dire autant des corrélations entre pourcentage de peau et d'os, ou de peau et de muscles, qui cependant ne sont significatives que chez les femelles, ainsi que de la relation négative dans les deux sexes entre pourcentage de sang et de plumes.

La corrélation entre l'importance des 2 tissus gras mesurés, graisse abdominale et graisse intermusculaire, est positive et sa valeur est relativement élevée. Ces 2 variables présentent une corrélation positive avec la peau (riche en lipides), significative sauf pour la graisse intermusculaire chez les mâles.

Les tissus gras présentent partout une corrélation négative avec le pourcentage de muscles; cela ne paraît pas résulter seulement du fait, pour les variables en cause, d'être des proportions d'un même total, car la corrélation n'est pas plus grande avec la graisse intermusculaire qu'avec la graisse abdominale. La relation est moins constante entre engraissement et pourcentage d'os. Les 2 tissus gras ont, par ailleurs, une corrélation fortement négative avec l'importance relative du gésier et, en ce qui concerne la graisse intermusculaire chez les femelles, avec le pourcentage de plumes.

Nous ne commenterons pas les autres corrélations significatives, dont une interprétation n'apparaît pas pour l'instant.

\section{Conclusion}

La valeur plus élevée des rendements en viande chez les oiseaux « Cou nu » provient de 3 sources : réduction du plumage, augmentation de la musculature et diminution du tissu gras dans la carcasse éviscérée. L'avantage moyen sur l'ensemble des 2 sexes, de l'ordre de 4 ou 3 p. 100 respectivement si l'on déduit ou non la graisse intermusculaire, paraît suffisant pour justifier des recherches ultérieures sur l'intérêt du gène $\mathrm{Na}$ dans la perspective d'une expansion de la production du poulet de découpe.

Reçu le 23 décembre 1983. 


\section{Références bibliographiques}

Bordas A., Mérat P., Sergent D, Ricard F.H., 1978. Influence du gène $N a$ (« Cou nu ») sur la croissance, la consommation alimentaire et la composition corporelle du poulet selon la température ambiante. Ann. Génét. Sél. Anim., 10, 209-231.

Chambers J.R., Fortin A., Grunder A.A., 1983. Relationships between carcass fatness and feed efficiency and its component traits in broiler chickens. Poult. Sci., 62, 2201-2207.

DelPeCh P., Ricard F., 1965. Relation entre les dépôts adipeux viscéraux et les lipides corporels chez le poulet. Ann. Zootech., 14, 181-189.

EHINGER F., 1977. Beziehungen zwischen Hautfett, gänzkörper und plasma Werten bei broilern verschiedener Herkunft. Arch. Geflügelkd., 41, 35-37.

Ehinger F., Seemann G., 1982. Einfluss von Futter, Alter und Geschlecht auf die Mastleistung und die Schlachtkörperqualität von Broilern verschiedener Herkunft. 2. Verfettungsgrad. Arch. Geflügelkd., 46, 177-188.

GoldsPinK G., 1977. The growth of muscles. In : Boorman K.N. \& Wilson B.J. (ed.), Growth and poultry meat production, Poultry Science Symposium n ${ }^{\circ} 12,13-28$, British Poultry Science.

HANZl C.J., Somes R.G. Jr., 1983. The effect of the Naked Neck gene, $\mathrm{Na}$, on growth and carcass composition of broilers raised in two temperatures. Poult. Sci., 62, 934-941.

Leclerce B., Blum J.C., BoYer J.P., 1980. Selecting broilers for low or high abdominal fat : initial observations. Br. Poult. Sci., 21, 107-113.

LIN C.Y., 1981. Relationships between increased body weight and fat deposition in broilers. World's Poult. Sci. J., 37, 106-110.

Mérat P., Bordas A., Ricard F.H., 1980. Composition anatomique, production d'œufs et efficacité alimentaire de poules pondeuses. Corrélations phénotypiques. Ann. Génét. Sél. Anim., 12, $191-200$.

VAN Middelkoop J.H., KUIT A.R., ZegWAARd A., 1977. Genetic factors in broiler fat deposition. In : Boorman K.N. \& Wilson B.J. (ed.), Growth and poultry meat production, Poultry Science Symposium $n^{\circ} 12,131-143$, British Poultry Science.

Monnet L.E., Bordas A., Mérat P., 1979. Gène Cou nu et performances de croissance selon la température chez le poulet. Ann. Génét. Sél. Anim., 11, 397-411.

RicARD F.H., 1972. Recherche d'une mesure précise de l'épaisseur de la peau chez le poulet. Ann. Zootech., 21, 479-493.

Ricaro F.H., 1968. Essais d'estimation de l'épaisseur et de l'importance de la peau chez le poulet. Ann. Zootech., 17, 459-466.

Ricard F.H., Rouvier R., 1967. Etude de la composition anatomique du poulet de chair. I. Variabilité de la répartition des différentes parties corporelles chez des coquelets Bresse pile. Ann. Zootech., 16, 23-29.

SOMES R.G. Jr., JoHNSON Susan, 1982. The effect of the scaleless gene, sc, on growth performance and carcass composition of broilers. Poult. Sci., 61, 414-423.

TOUCHBURn S., SimOn J., LeClerCo B., 1981. Evidence of a glucose-insulin imbalance and effect of dietary protein and energy level in chickens selected for high abdominal fat content. J. Nutr., 111, 325-335.

Zein-el-Dein A., Ayoub H., Mérat P., 1981a. Gène Cou nu et performances de croissance de poulets à deux saisons différentes en Egypte. Ann. Génét. Sél. Anim., 13, 269-280.

Zein-El-Dein A., Zeiny M., Ayoub H., 1981b. Carcass measurements of Naked Neck ans normal chicks. Ann. Génét. Sél. Anim., 13, 435-440.

Zein-El-Dein A., Bordas A., Mérat P., 1984. Croissance et efficacité alimentaire de poulets "Cou nu " ou normalement emplumés selon la teneur en protéines de la ration. Génét. Sél. Evol. (sous presse). 